\title{
Charged rotating dilaton black strings in AdS spaces
}

\author{
Ahmad Sheykhi* \\ Department of Physics, Shahid Bahonar University, P.O. Box 76175, Kerman, Iran \\ Research Institute for Astronomy and Astrophysics of Maragha (RIAAM), Maragha, Iran
}

We derive a class of charged rotating dilaton black string solutions in the background of anti-de-Sitter spaces with an appropriate combination of three Liouville-type dilaton potentials. We also present the suitable counterterm which removes the divergences of the action in the presence of dilaton potential. The solutions are analyzed and their thermodynamics is discussed by using the counterterm method.

There has been considerable attention in the past years in dilaton gravity. It is of great importance to investigate the effect of the dilaton field on the properties of the solutions. It was found that the dilaton field changes the causal structure of the black hole and leads to the curvature singularities at finite radii. In the absence of dilaton potential, exact solutions of charged dilaton black holes have been constructed by many authors [1, 2]. These black holes are all asymptotically flat. The presence of Liouville-type dilaton potential, which is regarded as the generalization of the cosmological constant, changes the asymptotic behavior of the solutions to be neither asymtotically flat nor (anti)-de Sitter [(A)dS]. Indeed, it has been shown that with the exception of a pure cosmological constant, no dilaton de-Sitter or anti-de-Sitter black hole solution exists with the presence of only one Liouville-type dilaton potential [3]. In the presence of one or two Liouvilletype potential, black hole spacetimes which are neither asymptotically flat nor (A)dS have been explored by many authors (see e.g. [4, 5, 6, 7]). Although these kind of solutions may shed some light on the possible extensions of AdS/CFT correspondence, they are physically less interesting due to their unusual asymptotic behavior.

On the other side, the construction and analysis of black hole solutions in (A)dS space is a subject of much recent interest. This is primarily due to their relevance for the AdS/CFT correspondence. It was argued that the thermodynamics of black holes in AdS spaces can be identified with that of a certain dual CFT in the high temperature limit [8]. Having the AdS/CFT correspondence idea at hand, one can gain some insights into thermodynamic properties and phase structures of strong 't Hooft coupling CFTs by studying thermodynamics of AdS black holes. Recently, the dilaton potential leading to (anti)-de Sitter-like solutions of dilaton gravity has been

\footnotetext{
*sheykhi@mail.uk.ac.ir
} 
found [9] (see also [10]). It was shown that the cosmological constant is coupled to the dilaton in a very nontrivial way. With the combination of three Liouville-type dilaton potentials, a class of static dilaton black hole solutions in (A)dS spaces has been obtained by using a coordinates transformation which recast the solution in the schwarzschild coordinates system [9]. The purpose of the present Letter is to construct a class of charged rotating dilaton black string solutions in the background of AdS spacetime. We will also present the suitable counterterm which removes the divergences of the action. Finally, we analyze the solutions and calculate their conserved and thermodynamic quantities by using the counterterm method inspired by AdS/CFT correspondence.

Our starting point is the four-dimensional Einstein-Maxwell-dilaton action

$$
\begin{aligned}
I_{G}= & -\frac{1}{16 \pi} \int_{\mathcal{M}} d^{4} x \sqrt{-g}\left(R-2 \partial_{\mu} \Phi \partial^{\mu} \Phi-V(\Phi)-e^{-2 \alpha \Phi} F_{\mu \nu} F^{\mu \nu}\right) \\
& -\frac{1}{8 \pi} \int_{\partial \mathcal{M}} d^{3} x \sqrt{-\gamma} \Theta(\gamma),
\end{aligned}
$$

where $R$ is the Ricci scalar curvature, $\Phi$ is the dilaton field, and $V(\Phi)$ is a potential for $\Phi$. $\alpha$ is a constant determining the strength of coupling of the scalar and electromagnetic field, $F_{\mu \nu}=$ $\partial_{\mu} A_{\nu}-\partial_{\nu} A_{\mu}$ is the electromagnetic field tensor and $A_{\mu}$ is the electromagnetic potential. The last term in Eq. (1) is the Gibbons-Hawking surface term. It is required for the variational principle to be well-defined. The factor $\Theta$ represents the trace of the extrinsic curvature for the boundary $\partial \mathcal{M}$ and $\gamma$ is the induced metric on the boundary. While $\alpha=0$ corresponds to the usual EinsteinMaxwell-scalar theory, $\alpha=1$ indicates the dilaton-electromagnetic coupling that appears in the low energy string action in Einstein's frame. In this Letter, we examine action (11) with three Liouville-type dilaton potentials $[9]$

$$
V(\Phi)=\frac{2 \Lambda}{3\left(\alpha^{2}+1\right)^{2}}\left[\alpha^{2}\left(3 \alpha^{2}-1\right) e^{-2 \Phi / \alpha}+\left(3-\alpha^{2}\right) e^{2 \alpha \Phi}+8 \alpha^{2} e^{\Phi(\alpha-1 / \alpha)}\right],
$$

where $\Lambda$ is the cosmological constant. It is clear the cosmological constant is coupled to the dilaton in a very nontrivial way. This type of dilaton potential can be obtained when a higher dimensional theory is compactified to four dimensions, including various supergravity models [11]. The equations of motion can be obtained by varying the action (11) with respect to the gravitational field $g_{\mu \nu}$, the dilaton field $\Phi$, and the gauge field $A_{\mu}$ which yields the following field equations

$$
\begin{gathered}
R_{\mu \nu}=2 \partial_{\mu} \Phi \partial_{\nu} \Phi+\frac{1}{2} g_{\mu \nu} V(\Phi)+2 e^{-2 \alpha \Phi}\left(F_{\mu \eta} F_{\nu}^{\eta}-\frac{1}{4} g_{\mu \nu} F_{\lambda \eta} F^{\lambda \eta}\right) \\
\nabla^{2} \Phi=\frac{1}{4} \frac{\partial V}{\partial \Phi}-\frac{\alpha}{2} e^{-2 \alpha \Phi} F_{\lambda \eta} F^{\lambda \eta}
\end{gathered}
$$




$$
\partial_{\mu}\left(\sqrt{-g} e^{-2 \alpha \Phi} F^{\mu \nu}\right)=0
$$

Our aim here is to construct charged rotating black string solutions of the field equations (3)-(5) and investigate their properties. The metric of four-dimensional rotating solution with cylindrical or toroidal horizons can be written as [12]

$$
\begin{aligned}
d s^{2} & =-f(r)(\Xi d t-a d \phi)^{2}+r^{2} R^{2}(r)\left(\frac{a}{l^{2}} d t-\Xi d \phi\right)^{2}+\frac{d r^{2}}{f(r)}+\frac{r^{2}}{l^{2}} R^{2}(r) d z^{2}, \\
\Xi^{2} & =1+\frac{a^{2}}{l^{2}},
\end{aligned}
$$

where $a$ is the rotation parameter. The functions $f(r)$ and $R(r)$ should be determined and $l$ has the dimension of length which is related to the constant $\Lambda$ by the relation $l^{2}=-3 / \Lambda$. The two dimensional space, $t=$ constant and $r=$ constant, can be (i) the flat torus model $T^{2}$ with topology $S^{1} \times S^{1}$, and $0 \leq \phi<2 \pi, 0 \leq z<2 \pi l$, (ii) the standard cylindrical model with topology $R \times S^{1}$, and $0 \leq \phi<2 \pi,-\infty<z<\infty$, and (iii) the infinite plane $R^{2}$ with $-\infty<\phi<\infty$ and $-\infty<z<\infty$. We will focus upon (i) and (ii). The Maxwell equation (5) can be integrated immediately to give

$$
\begin{aligned}
& F_{t r}=\frac{q \Xi e^{2 \alpha \Phi}}{r^{2} R^{2}(r)} \\
& F_{\phi r}=-\frac{a}{\Xi} F_{t r}
\end{aligned}
$$

where $q$, an integration constant, is related to the electric charge of black string. Inserting the Maxwell fields (7) and the metric (6) in the field equations (3) and (4), we can write these equation for $a=0$ as

$$
\begin{aligned}
& 2 r^{4} R^{3} R^{\prime} f^{\prime}+r^{4} R^{4} f^{\prime \prime}+2 r^{3} R^{4} f^{\prime}+r^{4} R^{4} V(\Phi)-2 q^{2} e^{2 \alpha \Phi}=0, \\
& 2 r^{3} R^{4} f^{\prime}+r^{4} R^{4} f^{\prime \prime}+8 r^{3} R^{3} f R^{\prime}+4 r^{4} R^{3} f R^{\prime \prime}+2 r^{4} R^{3} R^{\prime} f^{\prime} \\
& +4 r^{4} R^{4} f \Phi^{\prime 2}+r^{4} R^{4} V(\Phi)-2 q^{2} e^{2 \alpha \Phi}=0, \\
& 8 r^{3} R^{3} f R^{\prime}+2 r^{3} R^{4} f^{\prime}+2 r^{4} R^{3} f^{\prime} R^{\prime}+2 r^{2} R^{4} f+2 r^{4} R^{2} f R^{\prime 2} \\
& +2 r^{4} R^{3} f R^{\prime \prime}+r^{4} R^{4} V(\Phi)+2 q^{2} e^{2 \alpha \Phi}=0, \\
& r^{4} R^{4} \Phi^{\prime} f^{\prime}+r^{4} R^{4} \Phi^{\prime \prime} f+2 r^{3} R^{4} \Phi^{\prime} f+2 r^{4} R^{3} R^{\prime} \Phi^{\prime} f-r^{4} R^{4} \frac{\partial V}{4 \partial \Phi}-\alpha q^{2} e^{2 \alpha \Phi}=0,
\end{aligned}
$$

where the "prime" denotes differentiation with respect to $r$. Subtracting Eq. (9) from Eq. (8) gives

$$
2 R^{\prime}+r R^{\prime \prime}+r R \Phi^{\prime 2}=0
$$

Then we make the ansatz

$$
R(r)=e^{\alpha \Phi}
$$


Substituting this ansatz in Eq. (12), it reduces to

$$
r \alpha \Phi^{\prime \prime}+2 \alpha \Phi^{\prime}+r\left(1+\alpha^{2}\right) \Phi^{\prime 2}=0
$$

which has a solution of the form

$$
\Phi(r)=\frac{\alpha}{\alpha^{2}+1} \ln \left(1-\frac{b}{r}\right)
$$

where $b$ is an integration constants. Inserting (15), the ansatz (13), and the dilaton potential (2) into the field equations (8)-(11), one can show that these equations have the following solution

$$
f(r)=-\frac{c}{r}\left(1-\frac{b}{r}\right)^{\frac{1-\alpha^{2}}{1+\alpha^{2}}}-\frac{\Lambda}{3} r^{2}\left(1-\frac{b}{r}\right)^{\frac{2 \alpha^{2}}{\alpha^{2}+1}}
$$

where $c$ is an integration constant. The above solutions will fully satisfy the system of equations (8) (11) provided we have $q^{2}\left(1+\alpha^{2}\right)=b c$. One can also check that these solutions satisfy equations (3)-(5) in the rotating case where $a \neq 0$. It is apparent that this spacetime is asymptotically (anti)-de-Sitter. The Kretschmann scalar $R_{\mu \nu \lambda \kappa} R^{\mu \nu \lambda \kappa}$ and the Ricci scalar $R$ diverge at $r=0$ and therefore there is an essential singularity located at $r=0$. The explicit form of the Kretschmann scalar is complicated and we do not present it here, however, the Ricci scalar curvature of the metric has a simpler form and can be written as

$$
R=-\frac{2 q^{2} \alpha^{2} b\left(1-\frac{b}{r}\right)^{\frac{1-\alpha^{2}}{1+\alpha^{2}}}}{(r-b)^{2}\left(\alpha^{2}+1\right) r^{3}}+\frac{2 \Lambda\left(1-\frac{b}{r}\right)^{\frac{2 \alpha^{2}}{\alpha^{2}+1}}\left(2(r-b)^{2}+4 \alpha^{2} r(r-b)+2 \alpha^{4} r^{2}-\alpha^{2} b^{2}\right)}{\left(\alpha^{2}+1\right)^{2}(r-b)^{2}} .
$$

One can easily check that for the arbitrary values of the dilaton coupling constant, $R \rightarrow 4 \Lambda$ as $r \rightarrow \infty$. We also find out that for arbitrary $\alpha$ the Kretschmann scalar approach $8 \Lambda^{2} / 3$ as $r \rightarrow \infty$. This confirm our above discussion that our solution is asymptotically anti-de-Sitter. In the absence of a nontrivial dilaton $(\alpha=0)$, the solution reduces to the asymptotically (anti)-de-Sitter charged rotating black string [12]. However, for $\alpha \neq 0$ the solution is qualitatively different. As one can see from Eq. (17), the surface $r=b$ is a curvature singularity except for the case $\alpha=0$ when it is a nonsingular inner horizon. This is consistent with the idea that the inner horizon is unstable in the Einstein-Maxwell theory. Therefore, our solutions describe black strings in the case $b<r_{+}$, where $r_{+}$is the outer horizon of the black string (the root of Eq. $f(r)=0$ ). The above discussions will become more clear if we look on the figures 1 and 2, where we have plotted the function $f(r)$ versus $r$ for different values of the dilaton coupling $\alpha$ and the charge parameter $q$. In the case $r=b$, it is clear from (6) and (13) that for $\alpha \neq 0$ the area of the event horizon goes to zero (since $R(r) \rightarrow 0$ in this case). 


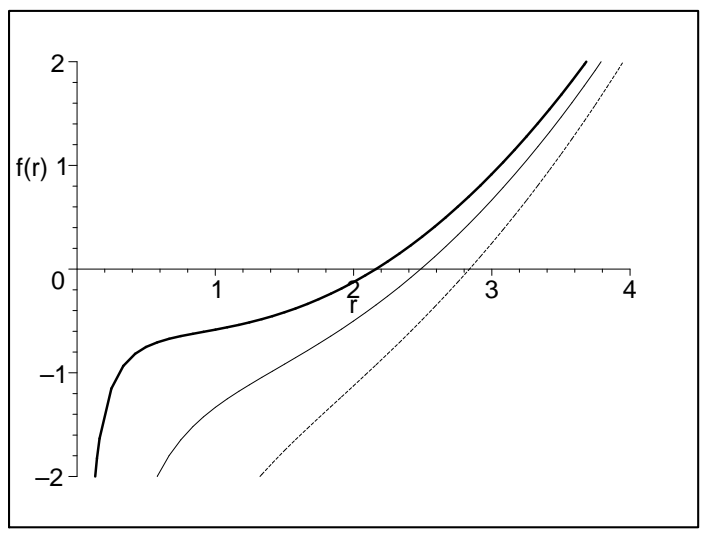

FIG. 1: The function $f(r)$ versus $r$ for $\alpha=1, b=2$ and $\Lambda=-1 . q=0.5$ (bold line), $q=1$ (continuous line) and $q=1.5$ (dashed line).

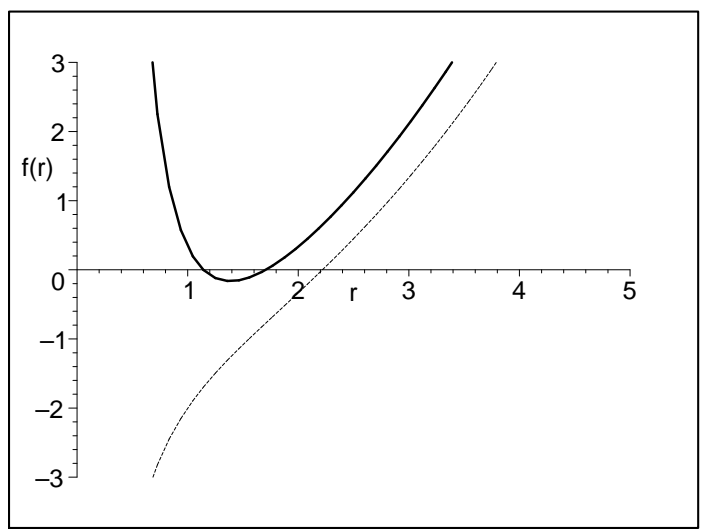

FIG. 2: The function $f(r)$ versus $r$ for $b=1, q=2$ and $\Lambda=-1$. $\alpha=0$ (bold line), $\alpha=1$ (dashed line).

In summary, compared to the charged black holes/strings in (A)dS universe, the dilaton version has some remarkable properties. In the first place, there may be three horizons in the ReissnerNordstrmde Sitter spacetime, i.e., black hole event horizon, black hole Cauchy horizon and cosmic event horizon. However, the charged dilaton black holes/strings in (A)dS spaces has at most two horizons. Here the inner Cauchy horizon disappears. This is due to the fact that the inner horizon is unstable, as pointed by Garfinkle [2].

Next, we calculate the conserved quantities of the solutions. For asymptotically (anti)-de-Sitter solutions, the way that one can calculate these quantities and obtain finite values for them is through the use of the counterterm method inspired by (A)dS/CFT correspondence [13]. In this paper we deal with the spacetimes with zero curvature boundary, $R_{a b c d}(\gamma)=0$, and therefore the counterterm for the stress energy tensor should be proportional to $\gamma^{a b}$. We find the suitable 
counterterm which removes the divergences in the form

$$
I_{c t}=-\frac{1}{8 \pi} \int_{\partial \mathcal{M}} d^{3} x \sqrt{-\gamma}\left(-\frac{1}{l}+\frac{\sqrt{-6 V(\Phi)}}{2}\right) .
$$

One may note that in the absence of a dilaton field where we have $V(\Phi)=2 \Lambda=-6 / l^{2}$, the above counterterm has the same form as in the case of asymptotically (A)dS solutions with zero-curvature boundary. Having the total finite action $I=I_{G}+I_{c t}$ at hand, one can use the quasilocal definition to construct a divergence free stress-energy tensor [14]. Thus the finite stress-energy tensor in four dimensional Einstein-dilaton gravity with three Liouville-type dilaton potentials (2) can be written as

$$
T^{a b}=\frac{1}{8 \pi}\left[\Theta^{a b}-\Theta \gamma^{a b}+\left(-\frac{1}{l}+\frac{\sqrt{-6 V(\Phi)}}{2}\right) \gamma^{a b}\right],
$$

The first two terms in Eq. (19) are the variation of the action (11) with respect to $\gamma_{a b}$, and the last two terms are the variation of the boundary counterterm (18) with respect to $\gamma_{a b}$. To compute the conserved charges of the spacetime, one should choose a spacelike surface $\mathcal{B}$ in $\partial \mathcal{M}$ with metric $\sigma_{i j}$, and write the boundary metric in ADM (Arnowitt-Deser-Misner) form:

$$
\gamma_{a b} d x^{a} d x^{a}=-N^{2} d t^{2}+\sigma_{i j}\left(d \varphi^{i}+V^{i} d t\right)\left(d \varphi^{j}+V^{j} d t\right)
$$

where the coordinates $\varphi^{i}$ are the angular variables parameterizing the hypersurface of constant $r$ around the origin, and $N$ and $V^{i}$ are the lapse and shift functions respectively. When there is a Killing vector field $\xi$ on the boundary, then the quasilocal conserved quantities associated with the stress tensors of Eq. (19) can be written as

$$
Q(\xi)=\int_{\mathcal{B}} d^{2} x \sqrt{\sigma} T_{a b} n^{a} \xi^{b}
$$

where $\sigma$ is the determinant of the metric $\sigma_{i j}, \xi$ and $n^{a}$ are, respectively, the Killing vector field and the unit normal vector on the boundary $\mathcal{B}$. For boundaries with timelike $(\xi=\partial / \partial t)$ and rotational $(\varsigma=\partial / \partial \varphi)$ Killing vector fields, one obtains the quasilocal mass and angular momentum

$$
\begin{aligned}
M & =\int_{\mathcal{B}} d^{2} x \sqrt{\sigma} T_{a b} n^{a} \xi^{b}, \\
J & =\int_{\mathcal{B}} d^{2} x \sqrt{\sigma} T_{a b} n^{a} \varsigma^{b} .
\end{aligned}
$$

These quantities are, respectively, the conserved mass and angular momenta of the system enclosed by the boundary $\mathcal{B}$. Note that they will both depend on the location of the boundary $\mathcal{B}$ in the spacetime, although each is independent of the particular choice of foliation $\mathcal{B}$ within the surface 
$\partial \mathcal{M}$. The mass and angular momentum per unit length of the string when the boundary $\mathcal{B}$ goes to infinity can be calculated through the use of Eqs. (21) and (22). We find

$$
\begin{gathered}
M=\frac{\alpha^{2}\left(\alpha^{2}-1\right) b^{3}}{24 \pi l^{3}\left(\alpha^{2}+1\right)^{3}}+\frac{\left(3 \Xi^{2}-1\right) c}{16 \pi l}, \\
J=\frac{3 \Xi c \sqrt{\Xi^{2}-1}}{16 \pi} .
\end{gathered}
$$

For $a=0(\Xi=1)$, the angular momentum per unit volume vanishes, and therefore $a$ is the rotational parameters of the spacetime. The entropy of the dilaton black string still obeys the so called area law of the entropy which states that the entropy of the black hole is a quarter of the event horizon area [15]. This near universal law applies to almost all kinds of black holes, including dilaton black holes, in Einstein gravity [16]. It is a matter of calculation to show that the entropy per unit length of the string is

$$
S=\frac{r_{+}^{2} \Xi\left(1-\frac{b}{r_{+}}\right)^{\frac{2 \alpha^{2}}{\alpha^{2}+1}}}{4 l} .
$$

By analytic continuation of the metric we can obtain the temperature and angular velocity of the horizon. The analytical continuation of the Lorentzian metric by $t \rightarrow i \tau$ and $a \rightarrow i a$ yields the Euclidean section, whose regularity at $r=r_{+}$requires that we should identify $\tau \sim \tau+\beta_{+}$and $\phi \sim \phi+i \beta_{+} \Omega_{+}$where $\beta_{+}$and $\Omega_{+}$are the inverse Hawking temperature and the angular velocity of the horizon. We find

$$
\begin{aligned}
& T_{+}=\frac{f^{\prime}\left(r_{+}\right)}{4 \pi \Xi}=\frac{\left(1-\frac{b}{r_{+}}\right)^{\frac{\alpha^{2}-1}{\alpha^{2}+1}}}{2 \pi r_{+}^{2} \Xi\left(\alpha^{2}+1\right)}\left(-\frac{\Lambda}{3} r_{+}^{2}\left(\alpha^{2} r_{+}+r_{+}-b\right)+\frac{c\left(\alpha^{2} r_{+}+r_{+}-2 b\right)}{2 r_{+}}\left(1-\frac{b}{r_{+}}\right)^{\frac{1-3 \alpha^{2}}{\alpha^{2}+1}}\right) \\
& \Omega_{+}=\frac{a}{\Xi l^{2}} .
\end{aligned}
$$

The next quantity we are going to calculate is the electric charge of the string. To determine the electric field we should consider the projections of the electromagnetic field tensor on special hypersurface. The normal vectors to such hypersurface are

$$
u^{0}=\frac{1}{N}, \quad u^{r}=0, \quad u^{i}=-\frac{V^{i}}{N}
$$

where $N$ and $V^{i}$ are the lapse function and shift vector. Then the electric field is $E^{\mu}=$ $g^{\mu \rho} e^{-2 \alpha \phi} F_{\rho \nu} u^{\nu}$, and the electric charge per unit length of the string can be found by calculating the flux of the electric field at infinity, yielding

$$
Q=\frac{\Xi q}{4 \pi l}
$$


The electric potential $U$, measured at infinity with respect to the horizon, is defined by [17]

$$
U=\left.A_{\mu} \chi^{\mu}\right|_{r \rightarrow \infty}-\left.A_{\mu} \chi^{\mu}\right|_{r=r_{+}},
$$

where $\chi=\partial_{t}+\Omega \partial_{\phi}$, is the null generator of the event horizon. One can easily show that the vector potential $A_{\mu}$ corresponding to the electromagnetic tensor (77) can be written as

$$
A_{\mu}=-\frac{q}{r}\left(\Xi \delta_{\mu}^{t}-a \delta_{\mu}^{\phi}\right) .
$$

Substituting (30) in (29) we obtain the electric potential as

$$
U=\frac{q}{\Xi r_{+}}
$$

Having the conserved and thermodynamic quantities of the rotating dilaton black string at hand, we are in a position to check the first law of black hole thermodynamics. Numerical calculations show that the conserved quantities calculated above satisfy the first law of thermodynamics

$$
d M=T d S+\Omega d J+U d Q
$$

In conclusion, we constructed a new class of charged rotating solutions in four-dimensional Einstein-Maxwell-dilaton gravity with cylindrical or toroidal horizons in the presence of dilaton potentials and investigate their properties. These solutions are asymptotically anti-de Sitter. The cosmological constant couples the dilaton field in a nontrivial way. The dilaton potential with respect to the cosmological constant includes three Liouville-type potentials. This is consistent

with the arguments in [3] that no (anti)-de-Sitter version of dilaton black holes exist with only one Liouville-type dilaton potential. We found the suitable counterterm which removes the divergences of the action in the presence of three Liouville-type dilaton potential. We also computed the conserved and thermodynamic quantities of the solutions by using the conterterm method and verified numerically that these quantities satisfy the first law of black hole thermodynamics.

\section{Acknowledgments}

This work has been supported financially by Research Institute for Astronomy and Astrophysics of Maragha, Iran.

[1] G. W. Gibbons and K. Maeda, Nucl. Phys. B298, 741 (1988);

T. Koikawa and M. Yoshimura, Phys. Lett. B189, 29 (1987);

D. Brill and J. Horowitz, ibid. B262, 437 (1991). 
[2] D. Garfinkle, G. T. Horowitz and A. Strominger, Phys. Rev. D 43, 3140 (1991);

R. Gregory and J. A. Harvey, ibid. 47, 2411 (1993);

M. Rakhmanov, ibid. 50, 5155 (1994);

G. T. Horowitz and A. Strominger, Nucl. Phys. B 360 (1991) 197.

[3] S. J. Poletti, D. L. Wiltshire, Phys. Rev. D 50 (1994) 7260 ;

S. J. Poletti, J. Twamley and D. L. Wiltshire, Phys. Rev. D 51 (1995) 5720;

S. Mignemi and D. L. Wiltshire, Phys. Rev. D 46 (1992) 1475.

[4] K. C. K. Chan, J. H. Horne and R. B. Mann, Nucl. Phys. B447, 441 (1995).

[5] R. G. Cai, J. Y. Ji and K. S. Soh, Phys. Rev D 57, 6547 (1998);

R. G. Cai and Y. Z. Zhang, ibid. 64, 104015 (2001).

[6] G. Clement, D. Gal'tsov and C. Leygnac, Phys. Rev. D 67, 024012 (2003);

G. Clement and C. Leygnac, ibid. 70, 084018 (2004).

[7] A. Sheykhi, M. H. Dehghani, N. Riazi, Phys. Rev. D 75, 044020 (2007);

A. Sheykhi, N. Riazi, Phys. Rev. D 75, 024021 (2007);

A. Sheykhi, Phys. Rev. D 76, 124025 (2007);

A. Sheykhi, Phys. Lett. B 662 (2008) 7.

[8] E. Witten, Adv. Theor. Math. Phys. 2, 505 (1998).

[9] C. J. Gao, S. N. Zhang, Phys. Rev. D 70 (2004) 124019.

[10] C. J. Gao, S. N. Zhang, Phys. Lett. B 605 (2005) 185 ;

C. J. Gao, S. N. Zhang, Phys. Lett. B 612 (2005) 127.

[11] S. B. Giddings, Phys. Rev. D 68 (2003) 026006;

E. Radu, D. H. Tchrakian, Class. Quant. Gravit. 22, 879 (2005).

[12] J. P. S. Lemos, Class. Quantum Gravit. 12, 1081 (1995);

J. P. S. Lemos, Phys. Lett. B 353, 46 (1995).

[13] J. Maldacena, Adv. Theor. Math. Phys., 2, 231 (1998);

E. Witten, Adv. Theor. Math. Phys., 2, 253 (1998);

O. Aharony, S. S. Gubser, J. Maldacena, H. Ooguri and Y. Oz, Phys. Rep. 323,183 (2000).

[14] J. D. Brown and J. W. York, Phys. Rev. D 47, 1407 (1993).

[15] J. D. Beckenstein, Phys. Rev. D 7, 2333 (1973);

G. W. Gibbons and S. W. Hawking, Phys. Rev. D 15, 2738 (1977).

[16] C. J. Hunter, Phys. Rev. D 59, 024009 (1998);

S. W. Hawking, C. J Hunter and D. N. Page, ibid. 59, 044033 (1999).

[17] M. Cvetic and S. S. Gubser, J. High Energy Phys. 04, 024 (1999);

M. M. Caldarelli, G. Cognola and D. Klemm, Class. Quant. Gravit. 17, 399 (2000). 